\title{
Christliche Anthropologie und die Herausforderungen der Postmoderne
}

Der Artikel widmet sich den von jeder christlichen Anthropologie vorauszusetzenden Einsichten und deren Infragestellung durch das postmoderne Denken.

\section{Eckpunkte einer christlichen Anthropologie}

Nach Robert Spaemann gibt es heute die Gefahr, dass der Mensch sich selbst nur noch als Täuschung der Natur, als „Anthropomorphismus" wahrnimmt. Daher stellt sich vor dem Hintergrund der evolutionistischen Deutung von Natur und Kosmos ganz besonders die Frage nach dem Menschen. Ist er nichts anderes als ein höherentwickeltes Tier? Sind sein Geist und Erkennen eine Emergenz aus materiellen Prozessen? Wie ist es um seine Einzigartigkeit und - theologisch - Gottzugehörigkeit bestellt?

\section{Die Sonderstellung des Menschen im Bereich des Lebendigen}

Der Mensch ist ein eigener Gesamtentwurf im Bereich der Lebewesen, mit Bios und Geist als Einheit von beidem. Dies soll kurz ausgeführt und begründet werden: 
Man hat gesagt: Das Tier ist Organismus - der Mensch hat einen Leib. Der große Unterschied liegt ich der Weise, wie dem Tier der Organismus und dem Menschen der Leib gegeben ist. Das Tier kann in seinem Wirken nichts anzielen, was nicht mit seiner artspezifischen Instinktorganisation korreliert. Weil seine Strebekräfte instinktgebunden sind, ist das Tier ganz und gar eingelassen in eine Umwelt. Seine Sinnesorgane und Glieder sind auf ein „Positionsfeld“ hingespannt, das seinem Vitalinteresse zugeordnet ist. Das Tier vollzieht nichts, was nicht Impuls des Organismus im Ganzen wäre und keinen biologischen Sinn besäße. Das „Tier steckt in sich selbst“, wie Pleßner sagte. Es ist ganz und gar beseelter Organismus und nichts darüber hinaus. Die tierische Innerlichkeit ist durch den Trieb determiniert. (Das sie bestimmende Telos ist nicht ein solches, das die tierische Subjektivität qualifiziert.) Diese (sc. die Subjektivität des Tieres) ist reflexionslos. Sie weiß nichts von ihrem zukünftigen Nichtsein und deshalb weiß sie auch nicht vom Sein. Das Tier ist auch total in seine Umwelt eingelassen. Es „konstituiert eine artspezifische Umwelt, in deren Mittelpunkt es sich selbst befindet".

Der Mensch dagegen hat nicht primär eine Umwelt, sondern ist von Welt umgeben. Er vermag vitale Bedeutsamkeitszusammenhänge zu transzendieren. Menschliche Personen haben ihr Sosein, ihre Vitalsphäre, ihre Natur mit ihren sinnlichen Strebekräften, ihren Leib. Sie sind nicht in ihre Weise, zu sein, versenkt. ${ }^{2}$ Daraus resultiert eine Indeterminiertheit des menschlichen Individuums im Hinblick auf seine organischen und vitalen Konditionen. ${ }^{3}$ Die Epigenetik lehrt uns seit etwa 20 Jahren noch mehr über die Offenheit des Organismus in Richtung auf erwerbbare

1 Vgl. R. Spaemann, Personen. Über den Unterschied zwischen „Jemand“ und „etwas", Stuttgart 1996, $196 f$.

2 Vgl. ebd.

3 Vgl. N. López Moratalia, Indeterminación Biológica y Alma Humana, in: A. Pérez de Laborda (Hg.), Sobre el Alma (Collectanea Matritensia 1), El Escorial 2005, 119-162. 
Eigenschaften, die die Aktivität oder Inaktivität seiner genetischen Codes habituell regulieren. Dies mag wohl neue Freiheitsspielräume im Hinblick auf die genetische Vorprägung eröffnen. ${ }^{4}$

Der Mensch ist nicht auf eine Umwelt fixiert, sondern vermag zu einem Gegenstand an sich zu gelangen. Zu dieser Hinordnung auf Gegenstände an sich gibt es beim Menschen ein anthropologisches Korrelat: Wir finden bei ihm keine tierische Biologie, der ein metabiologisches Prinzip zusätzlich aufgestockt wurde, sondern eine eigene, spezifisch menschliche Biologie, die ihre Eigenart in Bezug auf ein metabiologisches Prinzip, nämlich den Geist, besitzt. ${ }^{5}$ Von daher ergibt sich auch die Sonderstellung des menschlichen Leibes:

Die menschliche Hand z.B. ist nicht auf eine bestimmte Umwelt hin spezialisiert (wie etwa die Greifhand des Affen). Sie hat ihr Sein in einer nicht festgelegten, zu allen möglichen Werkzeugen hin offenen Lebendigkeit, als Glied des menschlichen Leibes. Sie ist in ihrer Formgebung auf die Objektwelt hin spezialisiert, nicht auf eine bestimmte Umwelt hin. Das mannigfache Werkzeug ist neben der Sprache das grundständigste Merkmal zur Unterscheidung des Menschen vom Tier, auch vom Primaten. Sie sind beide Ausdruck einer sachbezogenen Verhaltensweise zur Welt. Schon in der Embryonalentwicklung tritt die gegenüber dem Tierreich eigenständige Ausformung der menschlichen Hand hervor.

Im Vollzug der Bewegung kann der Mensch seine Glieder zu freier und symbolischer Kombination verwenden, woraus schöpferische Gestalten geschaffen werden. Das menschliche Gesicht ist eigens dafür geschaffen, dass es durch Mimik dem sinnhaften Ausdruck des Wortes dient. Arnold Gehlen ist der Nachweis zu danken, dass der Mensch schon in seinen Bewegungen „intelli-

4 Vgl. B. Kegel, Epigenetik. Wie unsere Erfahrungen vererbt werden, Köln ${ }^{4}$ 2018, 80-117; vgl. dazu auch P. Spork, Gesundheit ist kein Zufall. Wie das Leben unsere Gene prägt - die neuesten Erkenntnisse der Epigenetik, München ${ }^{3} 2019$.

5 Vgl. E. Blechschmidt, Wie beginnt das menschliche Leben? Vom Ei zum Embryo, Stein am Rhein, ${ }^{7} 2002,30-82$. 
gent" ist. Seine Antriebsstruktur ist spezifisch übertierisch und nicht erst seine geistige Intelligenz.

Daraus lässt sich folgende Definition für das menschliche Leiborgan gewinnen: Ein Leiborgan ist ein solches, für dessen Morphologie von Anfang an die Ausrichtung auf die Welt der Gegenstände mitbestimmend ist. Der Mensch ist das einzige Wesen, das einen Leib hat.

Und nun muss man sagen: Es gibt in Wirklichkeit kein Mittleres zwischen In-einer-Umwelt-Sein und dem In-der-Welt-Sein und folgerichtig auch keines zwischen Organismus und Leib, denn dieser ist von seiner Objektbezogenheit, eben von seinem In-der-Welt-Sein geprägt.

Es ist widersinnig, eine kontinuierlich aufsteigende Reihe von Lebensformen anzunehmen, bei der eine davon auf einmal anfinge, über sich zu verfügen und ein Wesen zu werden, das Ursprung seiner eigenen Akte ist. Da nun auch der menschliche Leib von der Ausrichtung auf die Objektwelt geprägt ist, so setzt seine Verwirklichung immer schon die Existenz eines geistbegabten Wesens voraus, kann also ebenso wenig durch kontinuierliche Entwicklung von unten aus den herrschenden Naturgesetzlichkeiten entstehen wie der Mensch selbst. Eine tierische Form kann nicht „sich“ zum Menschen entwickeln.

Daher können wir sagen: Der Mensch besitzt im Kosmos und unter den anderen Lebewesen eine Sonderstellung, ein „Plus“, das ihn über die nichtmenschliche Natur erhebt. Anders als die Pflanzen und Tiere ist die Schöpfung im Menschen zu einem seinsverstehenden Verhalten erwacht. In seinem Erkennen greift der Mensch auf die Wirklichkeit im Ganzen aus. Aufgrund seines Geistes ist er zur Durchlichtung der übrigen Schöpfung im Verstehen fähig. Und dieses Verstehen führt ihn auch zum Erfassen der Transzendenz und zur Fähigkeit der Liebe. ${ }^{6}$ Darum ist der Mensch ein Novum im Bereich des Lebendigen.

6 Vgl. dazu N. López Moratalia, Indeterminación biológica, 149-159. 
So bleibt, was die Entstehung des Menschen betrifft, nur der Hervorgang durch Schöpfung. Damit soll natürlich nicht verneint sein, dass der Mensch mit Geist und Leib Konditionen im Pflanzen- und Tierreich haben kann. Bauprinzipien, die auch die Tierwelt bestimmen, sind in ihm übernommen, aber so, dass sie zu einer neuen Gesamtgestalt werden.

\section{Geistige Intentionalität}

Der Aufstieg der Neurowissenschaft hat dazu beigetragen, die geistigen Akte, Erkennen und Wollen, als eine Emergenz des neuronalen Systems zu betrachten. ${ }^{7}$ Mit seiner geistigen Intention richtet der Mensch sich jedoch auf ein Erkenntnisobjekt oder ein zu erlangendes Gut aus. Sollte es wahr sein, dass einem jeden solchen Objekt ein jeweils eindeutiger Gehirnzustand entspricht, wie die heutigen Neurowissenschaften behaupten, dann müsste es - so Robert Spaemann - möglich sein, ,aus dem Gehirn eines Mathematikers den Inhalt der Mathematik und aus dem des Historiker die Geschichte des Dreißigjährigen Krieges zu erschließen." ${ }^{\text {" }}$

Ohne die Ausrichtung auf ein Objekt der Erkenntnis oder des Handelns tritt die Eigenart des Geistigen überhaupt nicht in den Blick. Wenn man "Geist" auf individuell erlebbare Zustände einschränkt, damit er mit empirischen Methoden untersucht werden kann, „kommt man bestenfalls an das aktuale Selbstbewusstsein heran, nicht aber an die Spezifik geistige Phänomene “. ${ }^{9}$ Für die von Gerhard Roth u. a. vertretene Position

7 Vgl. M. Stickelbroeck, Das cerebrale Subjekt. Anfragen an eine Neurophilosophie, in: J. Kreiml / M. Stickelbroeck (Hg.), Die Person - ihr Selbstsein und ihr Handeln. Zur Philosophie Robert Spaemanns, Regensburg 2016, 180-212.

8 R. Spaemann, Personen. Versuche über den Unterschied zwischen "etwas" und "jemand", Stuttgart 1996, 59.

9 Vgl. H.-P. Krüger, Das Gehirn im Kontext exzentrischer Positionierungen. Zur philosophischen Herausforderung der neurobiologischen Hirnforschung, in: ders. (Hg.), Hirn als Subjekt, 61-98, 78. 
wird die mentale Sphäre zu einer Funktion der physischen; dies setzt allerdings voraus, dass ,sie nicht bereits durch den intentionalen Bezug auf physische Ereignisse definiert ist". ${ }^{10}$

Freilich gibt es eine Reihe von nicht intentionalen seelischen Zuständen wie z. B. Schmerzen, Stimmungen, Erregungszustände, die ohne einen solchen intentionalen Bezug zu einem physischen Objekt beschrieben werden können. Sie sind „als die subjektive Seite objektiver physischer Prozesse " zu deuten. ${ }^{11}$ Für die Differenz zwischen erlebbaren Zuständen wie Schmerzen und den ihnen zugrunde liegenden Begebenheiten ist eine dualistische Beschreibung angemessen, denn beides liegt auf einer anderen semantischen Ebene. ${ }^{12}$ Das medizinische Faktum einer Muskelprellung und der Schmerz, den sie mir versursacht, sind voneinander verschieden.

Wo es aber um Einstellungen geht, die eine Objektbezogenheit aufweisen und die anderen kommunizierbar sein sollen, kommt immer Intentionalität ins Spiel, die über solche unbezügliche Zustände hinausgeht. Die empirische Einlösung argumentativer Geltungsansprüche wird auch von der Hirnforschung intendiert, diese sind aber von anderer Ordnung als aktuale Bewusstseinszustände. Wäre dem nicht so, dann würden wir uns mit allen Behauptungen in der Sphäre des bloß Mentalen bewegen, die vom Physikalismus aber zuvor für ontologisch bedeutungslos erklärt wurde. Daher muss man eine Differenz zwischen psychischen Zuständen und intentionalen Akten einführen. ${ }^{13}$

Was die nichtintentionalen Zustände betrifft, so bleibt ihr ontologischer Status ambivalent. Die These, sie seien nicht etwas, was „es gibt“, kann zunächst widerspruchsfrei festgehalten werden. Anders verhält es sich jedoch, wenn es um intentionale Einstellungen geht: Alle jene Akte des Erkennens, Meinens, Be-

\footnotetext{
10 Vgl. R. Spaemann, Personen, 59.

${ }^{11}$ Vgl. ebd., 60.

12 Vgl. ebd., 60f.

${ }^{13}$ Vgl. ebd., 62.
} 
urteilens und Wollens richten uns aus auf eine Welt mit ihren materiellen Wesen, die aber nur in solchen Akten für uns objektiv wird. Wären sie bloß die subjektive Seite „an sich gleichgültiger neuronaler Prozesse", führte dies zur Vergleichgültigung der These, mit der dies behauptet wird. Es käme überhaupt auf nichts an, auch nicht auf die Differenz von wahren und falschen Urteilen. So geraten Roth u.a., die geistige Akte als neuronal induziert erklären, in einen performativen Selbstwiderspruch, da sie ja schließlich einen Geltungsanspruch etablieren und diesen anderen kommunizieren wollen. Diese reductio ad absurdum führt R. Spaemann zu der Aussage:

„Wo also theoretische oder praktische Intentionalität ins Spiel kommt, da wird der materialistische Reduktionismus selbstwidersprüchlich. Intentionalität kann überhaupt nicht adäquat als psychischer Zustand beschreiben werden. ${ }^{\text {"14 }}$

\section{Der Mensch als mit einer Natur ausgerüstete Leib-Geist-Person}

Grundlegend ist die aus der Schöpfungslehre gewonnene Einsicht, dass der Mensch und sein Leben gänzlich von Gott abhängig sind. Schon aus den biblischen Texten spricht die Anerkennung der unendlichen Überlegenheit des Schöpfers, der sich endliche personale Freiheit gegenübersetzt, um von dort her die Rückantwort der Liebe zu empfangen. Der Mensch besitzt - verglichen mit der absoluten Seinsfülle Gottes - einen schwachen Seinsstand. Zwischen Gott und Mensch waltet eine unendliche Differenz.

Trotzdem stellt das Merkmal der Kreatürlichkeit nichts Negatives dar. Die scheinbare Nichtigkeit ergibt sich nur im Vergleich mit dem Schöpfer. Die mit dem Kreaturbewusstsein gegebene Begrenztheit ist nicht als etwas in sich Minderwertiges und Niedriges anzusehen. Der positive Aspekt wird schon bei der fei-

14 Ebd. 
erlichen „Selbstentschließung“ Gottes sichtbar: „Lasst uns den Menschen machen" (Gen 1,26). Darin zeigt sich schon die intensivere Anteilnahme Jahwes an diesem Werk, in dem die Stufenfolge der Geschöpfe ihren Gipfel erreicht. Der Mensch wird als einziges Wesen zur Unmittelbarkeit mit Gott erhoben.

\section{Personalität}

Gott verleiht in seinem liebenden Wollen allen Dingen ihre Individualität, ihre Besonderheit: er erschafft diese Pflanze, dieses Tier. Dies gilt im Besonderen - und in höherer Weise - auch für den Menschen. Er will diesen konkreten Menschen in seiner ureigenen Charakteristik. Jeder Mensch ist so innerhalb der Menschheit ein unwiederholbares Individuum. Jeder Mensch besitzt eine unverwechselbare Individualität, die nicht kopierbar ist. Es gibt kein anderes Wesen in der Schöpfung, das dieselbe Einzigartigkeit mit dem einzelnen konkreten Menschen teilt. Darum haben manche von der Nicht-Mitteilbarkeit des Personseins (Duns Scotus) gesprochen.

Vom ersten Augenblick seines Daseins an ist der Mensch diese Person - allein aufgrund seiner Existenz, die auf dem liebenden Gewollt-Sein in Gottes Wort beruht. Auf diesem Personsein sind vier bedeutende Charakteristika des Menschseins aufgebaut: die Autonomie (als Selbststand), die Selbsterkenntnis, die Verantwortlichkeit und die Selbsttranszendenz.

Die ureigene Einzigkeit eines jeden Menschen verleiht ihm im Gegenüber der anderen Menschen eine gewisse Autonomie, einen gewissen Selbststand, ein Stehen in sich Selbst. Daran angelehnt ist die Selbsterkenntnis. Die fußt freilich auf der Substantialität seines naturhaften Seins, die sich in den geistigen Vollzügen entfaltet. ${ }^{15}$ In seiner Einzigartigkeit erkennt der

15 So wurde bei Thomas von Aquin die von Natur aus bestehende Substanz (in Anlehnung an die próte ousía bei Aristoteles) als dasjenige bedacht, das nicht von einer anderen Sache ausgesagt werden oder in einem 
Mensch sich selbst im Lichte des je anderen Menschen. Im Blick auf die Unwiederholbarkeit des anderen Menschen erkennt der Mensch sein ureigenes So-Sein. Erst wenn diese Selbsterkenntnis zum Selbststand, zum Stehen in seinem ureigenen Sein wird, kann der Mensch seine Selbständigkeit und sein ureigenes Personsein verwirklichen. Darin liegt nun gerade die Verantwortung des einzelnen Menschen, sich dem Anderssein des anderen zu stellen, um so sein persönliches Sein zu entdecken und zu verwirklichen.

Personalität verweist den Menschen aber nicht nur auf den Anderen, es impliziert immer auch ein letztes Auf-sich-selbstVerwiesensein, in dem der Mensch sich in diesem ureigenen Selbstsein nicht nur als einzig, sondern auch als allein erfährt. An diesem Punkt wird Personalität zum Ort der Begegnung mit dem Schöpfer selbst, der in seiner Liebe diese Einzigkeit gewollt und angenommen hat.

Daher sind Autonomie und Selbststand und die daraus notwendig erforderliche Selbstverwirklichung des ureigenen Soseins sowohl in der Begegnung mit dem Anderen als auch in der letzten Selbstverwiesenheit auf die Selbsttranszendenz hin angelegt. Denn erst, wenn er Schritte über sich hinaus macht, erhält das eigene Sosein seine wahre bereichernde Bedeutung, und erst in der sich selbst bejahenden Begegnung mit dem ganz und vollkommen Anderen, Gott, erfährt der Mensch sich ganzheitlich liebend angenommen und geschenkt. Der Mensch ist folglich berufen, sich selbst erkennend aus sich herauszugehen und sein wahres Sein in der Beziehung zum anderen und zu Gott zu entfalten.

anderen Ding bestehen kann. Aristoteles hatte vom tode ti gesprochen, das nur mehr deiktisch angewiesen werden kann, indem man darauf hinzeigt. Insbesondere erfährt sich der Mensch in der Sprache als „first Person" - in solcher Identität mit sich selbst, die nicht medial besteht und die man auch nicht auf andere Weise deklinieren kann. 
Auf der Offenbarung der Schöpfung fußt die ganze theologische Anthropologie. Diese kommt aber erst im Hinübergehen in die Christologie zur vollen Bedeutung:

Zum einen bedeutet dies, dass die großen christologischen Grundaussagen, etwa die Inkarnation, die biblische Anthropologie voraussetzen: Die Inkarnation ist bei einem rein platonischen Verständnis des Menschen so unbegreiflich wie Kreuz und Auferstehung. Sie setzt das Jasagen zur Einheit des Menschen als einzigen und ungeteilten Wesens voraus. Die Hervorhebung des Individuums Mensch gipfelt im Gott-Menschen Jesus Christus. In ihm, durch ihn und auf ihn hin sind alle Menschen in ihrer je eigenen Individualität angenommen und gewollt (vgl. Kol 1,15-17). Die Inkarnation ist die höchste Realisierung des Gedankens, dass der ganze, leibhaftige Mensch Gottes Geschöpf ist. So dürfen wir sagen, dass die Inkarnation eine Grundlinie der biblischen Schöpfungstheologie vollendet, das Ja zum Menschen als Ganzheit, der im Leib Mensch und als solcher das Gegenüber Gottes ist.

Das gleiche gilt von der Auferstehung. Nur von Christus her ist begreiflich, dass die christliche Anthropologie in die Hoffnung der Auferstehung einmündet. So eröffnet sich auch ein eschatologischer Ausblick: Durch die Hineinnahme des Menschen in die Inkarnation und Auferstehung hat die Materie eine neue $\mathrm{Zu}$ kunft: sie wird nicht einfach zurückgelassen, wenn der Mensch vollendet ins Heil kommt, sondern nimmt Teil an der Verklärung des ganzen Menschen, die auf ihn wartet.

\section{Die Anthropologie vor den Herausforderungen der Postmoderne}

Unter den Theologen nehmen die Lobredner der Moderne meist nicht wahr, dass sich das neuzeitliche Denken einem Zustand genähert hat, in dem die ursprünglichen Werte der Aufklärung 
sich ins Gegenteil verkehren: An die Stelle der neuzeitlichen starken Subjektivität tritt ein schwärmerisches kosmisches Einheitsbewusstsein, an die Stelle der Ratio tritt der Mythos östlicher Prägung, an die Stelle des Vernunftglaubens (Kant) eine vagabundierende esoterische Religiosität, an die Stelle der menschlichen Freiheit und Verantwortung die Determination durch die kosmische Dynamik, in die der Mensch sich als in das Göttliche einschwingen muss. Dies muss zu desaströsen Konsequenzen in der Anthropologie führen.

\section{Postmodernes Denken in der Philosophie}

Wir haben es in der Postmoderne mit einer Abschaffung der Logos-Tradition zu tun. Auf philosophischer Ebene wären einige Positionen anzufragen, die von den neueren Theologien bisweilen zugrunde gelegt werden. ${ }^{16}$

In der Philosophie der Poststrukturalisten wird der epistemologische Relativismus legitimiert durch die normative Bejahung des Pluralismus. Es wäre angebracht, diese Epistemologie zunächst auf der Ebene philosophischer Argumentation zu kritisie-

${ }^{16}$ Für die Postmodernen gehört eine universale verbindliche Ethik zu den "großen Erzählungen“, die obsolet geworden sind. Die Postmoderne, die sich gegen die Obsessionen der Moderne stellt, bleibt dieser aber selbst verhaftet. Vgl. J. Estermann, Filosofía Andina: Sabiduría indígena para un mundo nuevo, La Paz 2006, 30:ttgt „La mísma filosofía postmoderna puede ser interpretada como la última expresión (dialéctica) del espíritu moderno occidental, pero de ninguna manera como la superación definitiva del paradigma occidental como tal (...) El esteticismo postmoderno enfatiza la conversión de valores cognitivos, éticos y sociales en valores netamente estéticos de agrado o desagrado." Estermann beklagt die desaströsen Folgen des Postmodernismus für die Ethik, bekennt sich allerdings selbst zur uneingeschränkten Diversität der Diskurse und „Symbolsysteme“. Insofern scheint mir der paradigmatische Kulturrelativismus bei ihm nicht konsequent zu Ende geführt. In der äquivalenten Gültigkeit aller kulturellen Wertungen verliert sich nämlich die universale Normativität des Ethischen. 
ren: Es können mehrere Positionen durchaus gleichzeitig wahr sein, vorausgesetzt, dass sie einander nicht widersprechen. Darum schließt das Festhalten am objektiven Wahrheitsbegriff eine Pluralität miteinander kompatibler Positionen keineswegs aus. Postmoderne Philosophen dispensieren sich allerdings davon.

Michael Foucault und Richard Rorty - die prominentesten Vertreter der postmodernen "Erkenntnistheorie“ - behaupten, dass Vernunft und Wahrheit prinzipiell pluralistisch verfasst seien. Ohne die von Foucault vollzogene Aufweichung des Wissensbegriffs lässt sich auch die „postmoderne Kondition“ Lyotards nicht denken: Durch die Erhebung eines Partikularen zum Universalen habe die Moderne Tyrannei und Terror erzeugt. Dieser Terror soll durch die sprachphilosophisch und erkenntnis-theoretisch begründete Einsicht, dass sich die Wahrheit nie eindeutig erfassen lässt, vom prinzipiellen Pluralismus der Postmoderne überwunden werden. ${ }^{17}$ Das Projekt der „Moderne“ ist für ihn gescheitert.

In philosophischer Hinsicht entscheidend und auch problematisch ist die von Lyotard angenommene strikte Verbindung von „Wissen“ und „Macht“, mit der er - über Nietzsche - auf eine Maxime der frühen Neuzeit zurückgreift, die jedoch in dieser Einseitigkeit weder für die „Modernen“ noch für die Tradition maßgebend ist. In der Frage nach der „Legitimation“, die für Lyotard nicht nur die Anleitung für die Kritik an der Moderne darstellt, sondern - unter dem Stichwort „Delegitimierung“ - den Weg zur veränderten, postmodernen Modellierung des Wissens weist, zeigt sich die Brisanz dieser Verbindung. In Folge einer radikalen Historisierung des Denkens wird eine Dekonstruktion und Auflösung des universalen Begriffs der Wahrheit, der mehr als momentane Gültigkeit besitzt, vollzogen.

Die Kehre, die in die Postmoderne führt, besteht darin, den Meta-Erzählungen von Freiheit, von Fortschritt keinen Glauben

17 Vgl. J.-F. Lyotard, Das postmoderne Wissen (franz.: la condition postmoderne), Wien ${ }^{7} 2012$, 14-16; 176; $189 f$. 
mehr zu schenken. Die „großen Erzählungen“ müssten aufgegeben werden. An ihre Stelle trete eine Vielfalt von Diskursen, die mit je eigenen Regeln der Konstitution und Verknüpfung von Aussagen folgen und mit eigenen Kriterien der Rationalität und Normativität einhergehen können. Lyotard beschrieb diese Diskurse als isolierte „Sprachspiele“. Es gehe darum, die "Widerstreite" ausfindig zu machen und die unterschiedlichen Positionen hervorzuheben, nicht aber, diese Dissense in einen Konsens zu überführen oder zu versöhnen. ${ }^{18}$ Da Lyotard das Wissen ausgehend von der "Pragmatik“ bestimmt, kommt es zu einer postmodernen Pluralität, ja zu einer Agonie der Lebensformen: Der Widerstreit bleibt prinzipiell unentscheidbar. Die Weise, in der Lyotard versucht, die „Idee und Praxis der Gerechtigkeit" einzulösen, läuft auf eine Art Schadensbegrenzung hinaus, wenn er fordert, dass kein einziger Diskurs und kein Weltbild eine Hegemonie über die andere beanspruchen darf. Es würde darum gehen, den Widerstreit zu erfahren, auszuhalten, ohne andere Arten von Diskurs zu verdrängen. Es ist wohl dem Umstand zu danken, dass es Lyotard gelungen ist, eine sehr komplexe Entwicklung zu durchschauen und mit begrifflichen Mitteln zu präzisieren, wenn er heute einen herausragenden Platz in der Diskussion einnimmt.

Bei den Postmodernen findet eine Abkehr vom Begründungsdenken statt. ${ }^{19}$ Vor allem bei Jacques Derrida begegnet die Figur eines unendlichen Regresses: In der Sprache will er keine unhintergehbare Struktur entdecken. Sie wird zum Garanten einer unendlichen Zeichenbeziehung ohne letzten Grund. Für jene,

18 Vgl. ders., Der Widerstreit, München 1987, 32-36; $236 f$.

19 Vgl. S. Sim, Postmodernism and Philosophy, in: ders. (Hg.), The Routledge Companion to Postmodernism, London 2001, 3-14. Vgl. auch B. Goebel und F. Suárez Müller, Postmodermismus: Status quo einer philosophischen Strömung, in: dies. (Hg.), Kritik der postmodernen Vernunft. Über Derrida, Foucault und andere zeitgenössische Denker, Darmstadt 2007, Einleitung, 8-28. 
die sich der Dekonstruktion verschrieben haben, dazu gehören vor allem Michel Foucault, Jacques Derrida, Francois Lyotard, Gianni Vattimo, Richard Rorty, spielt ein psychologischer „Energismus" eine quasi-metaphysische Erklärungsrolle für den Relativismus. Jenseits einer vitalistischen Vorstellung, die einer Naturalisierung rationaler Begründungsstrukturen gleichkommt, gibt es keine Wahrheiten, die allgemein-verbindlich wären. Niemand kommt an die wahre Realität, gerade auch die Realität des Menschen, heran, wenn alles nur „Interpretation“ ist.

Die Postmodernen selbst können der radikalen Historisierung des Denkens, die eine Dekonstruktion und Auflösung des universalen Begriffs der Wahrheit nach sich zieht, nicht entkommen. Weil sie selbst ihrerseits der Geschichte des Denkens angehören, geraten sie in unaufhebbare Selbstwidersprüche. ${ }^{20}$

Im Zuge der philosophischen Postmoderne hat sich ein betont anti-essentialistisches Denken breit gemacht, das den Dingen, auch dem Menschen, ein Wesen abspricht.

\section{2. Übergriff auf den Status des Menschen}

Der Postmodernismus verwirft Universalien, Essenzen und Genres ebenso wie die Idee der Einheit des Logos - als der Wahrheit und der Vernunft - sowie der Einheit des Menschen - nämlich seines in der traditionellen philosophischen (und theologischen) Anthropologie vorausgesetzten universalen Wesens.

Wo der Mensch nicht mehr in seiner naturhaften Substantialität und geistigen Intentionalität verstanden wird, löst sich jede klare Abgrenzung zur Tierwelt auf. Es gibt nur noch einen - in der Komplexität der neuronalen Verbindungen gelegenen graduellen Unterschied zu nichtmenschlichen Lebewesen. Wir befinden uns in einem Tier-Mensch-Übergangsfeld.

20 Darauf hat nicht zuletzt Thomas Nagl aufmerksam gemacht. Vgl. ders., Das letzte Wort, Stuttgart 1999, 22-56. 
Die im ersten Abschnitt vorgetragenen Überlegungen setzten mit der Rede von einem Wesen und einer Identität des Menschen ein Subjekt voraus, das immer schon ontologisch determiniert ist. Mit seinem konsequenten Anti-Essentialismus, der eine Wesensbestimmtheit des Menschen ablehnt, verliert der Postmodernismus jedes kritische Potential seiner Theorie. Er kann nur noch versuchen, im Rahmen einer Kulturgeschichte das Geflecht aus Theorien, Macht und Normen zu beschreiben, das zum Beispiel in der modernen biopolitique zum Ausdruck kommt. ${ }^{21}$

Der Traum von der unbändigen Freiheit des „deregulierten Subjekts" führt dazu, einem biotechnologischen Transhumanismus ${ }^{22}$ das Wort zu reden, in dem sich der substanzlose Mensch mit einem technischen „Kleid“ ausstattet oder sich selbst stets neu entwirft. ${ }^{23}$

${ }^{21}$ Vgl. M. Foucault, Naissance de la biopolitique. Cours au Collège de France (1978-1979), Paris 2004, 295. Bei Foucault wird das menschliche Wesen als wirtschaftliche Konstruktion gedacht.

${ }^{22}$ Vgl. C. Helmus, Transhumanismus - der neue (Unter-) Gang des Menschen? Das Menschenbild des Transhumanismus und seine Herausforderung für die Theologische Anthropologie, Regensburg 2020.

${ }^{23}$ Vgl. dazu M. Stickelbroeck, Zur Ontologie der Artefakte. Die kategoriale Analyse der Artefakte bei Aristoteles / Thomas von Aquin und ihre hermeneutische Relevanz, in: FTPh 65 (2018) 373-400. Nach der scharfsinnigen Analyse von Holm Tetens, lanciert die wissenschaftlich-technische Zivilisation, um den existentiell deprimierenden Konsequenzen ihres ersten Postulates - nämlich dem vom exklusiven Zugang der Wissenschaften zur Wirklichkeit) - zu entgehen, ein zweites Postulat, dem der Weltperfektionierung: „Es beinhaltet: Indem wir die Ergebnisse wissenschaftlicher Welterkenntnis technologisch anwnden und unter Bedingungen kapitalistischer Produktion von Waren und Dienstleistungen ökonomisch nutzen, schaffen wir eine immer bessere Welt, in der immer mehr Übel und Leiden aus ihr verschwinden." Vgl. H. Tetens, Der Glaube an die Wissenschaften und der methodische Atheismus. Zur religiösen Dialektik der wissenschaftlich-technischen Zivilisation, in: NZST 55 (2013) 271-283, hier: 273. So geht eben auch der neue, „ganze“ Mensch aus dem inventiven Eingriff in seine Natur hervor. 
So dämmert die Ära der Cyborgs herauf. ${ }^{24}$ Die Technikvisionen der 90er Jahre scheinen die unangenehmen Eigenschaften zu haben, sich in die Realität umsetzen zu lassen. Nathan S. Line und Manfred E. Clynes hatten bereits 1960 vorgeschlagen, den menschlichen Körper mit spezifischen Implantaten fit für das Leben im Weltraum zu machen. Seither lässt die inventive Vorstellung eines Mensch-Maschinen-Systems oder kybernetischer Organismen die neue Generation der Techniker nicht mehr los: Entgrenzung des menschlichen Körpers durch Technologie ist das Ziel - ein „Über-Hinaus“ des Menschen. Die Interferenz von Naturwesen Mensch und technischen Apparaten, die in seine Biologie integriert werden, scheint dem Individuum kurzfristig mehr Flexibilität in Raum und Zeit zu geben. Wird jedoch das dergestalt seiner Umwelt flexibler adaptierte und aufgerüstete Wesen dabei nicht in neue Systeme eingezwängt, die auch neue Abhängigkeiten mit sich bringen?

Man hat schon früh begonnen, fehlende Teile des menschlichen Körpers durch technische Elaborate auszugleichen. Sie entsprechen in ihrer Funktion dem, wozu der jeweilige Körperteil dienlich wäre, falls vorhanden. Dies scheint unproblematisch, solange der Mensch selbst es ist, der damit Tätigkeiten vollzieht, die seiner species entsprechen und der Verwirklichung seiner Vermögen dient: Künstliches Hüftgelenk, künstliche Knie, künstliche Hand, Stents in den Blutgefäßen... - aber Cyborgs sind dann doch etwas anderes.

Eine Grenzüberschreitung liegt dann vor, wenn die "Natur" (bei Aristoteles die prôte ousia) durch das technische Implantat oder durch genetische Manipulation derart verändert wird, das ein Mischwesen aus biologischer Substanz und Technologie (Mensch und Maschine) das Lab des Erfinders verlässt (so wie die „Borgs" in der Serie "Star-Trek“ auf einmal die Szenerie bevölkerten). Dabei findet in der Interferenz von menschlicher

${ }^{24}$ Vgl. D. Haraway, Simians, Cyborgs and Women: The Reinvention of Nature, London 1991, hier bes. 149 (Cyborg Manifesto). 
Erstform und technischen Komponenten eine Verlagerung der Gewichtung hin zur Maschine statt. ${ }^{25}$

In dem Fall wird vergessen, dass der Mensch, wie er von $\mathrm{Na}-$ tur her da ist, die Grundlage dafür bietet, dass Technik überhaupt sein kann, während diese in ihrer vom Menschen gesetzten Zweckbestimmung (externe Teleologie) aufgeht. Prima facie erfolgt die Integration von Mensch und Maschine zwar freiwillig, um die Anpassung an neue Umgebungen zu ermöglichen; sie läuft dann aber unbewusst weiter, ohne die personalen Entscheidungen zu beachten, mit denen der Mensch er selbst bleiben kann.

Wo Technologie auf den Menschen selbst angewandt wird, um in seine Biologie integriert zu werden, gilt es - um des Menschen willen - auf der Hut zu sein: Die Technik, die durch bestimmte Bedürfnisse entstanden ist und zur Kompensation seiner fehlenden oder defizienten Ausstattung bzw. zur Organentlastung dient, greift gravierend in die menschlichen Handlungsfelder ein - bereits dort, wo sie seinem Organismus äußerlich bleibt. Sie wirkt schon bei der permanenten Nutzung von Apparaten auf den zurück, der sie gebraucht, und führt zu Kompetenzverlust und zur Änderung seiner Denkformen und seines Handelns. (Schon vor dem Aufkommen der super-think-tanks des Silicon Valley). ${ }^{26}$

25 Vgl. G.S. Freyermuth, Übermenschenbilder. Visionen von Cyborgs künden von einer neuen Kultur menschlicher Selbstverbesserung, in: „Spektrum der Wissenschaft Spezial“ 2/15 (Mensch, Maschine, Visionen. Wie Biologie und Technik verschmelzen) 57-65. Die Mitglieder des amerikanischen President's Council for Bioethics verfassten 2003 eine 300 Seiten starke Studie "Beyond Therapy. Biotechnology and the Pursuit of Happiness" (New York: Publisher. Dana Press 2003), in der sie davor warnen, dass die Technisierung des Körpers unser gesamtes Welt- und Menschenbild auf den Kopf stellen würde.

${ }^{26}$ Vgl. T. Krämer, Kommt die gesteuerte Persönlichkeit?, in: „Spektrum der Wissenschaft Spezial“" 2/15, 40-47. 
Elan Musk, Tesla-Gründer und Multimilliardär, möchte mit seinem 2016 gegründeten Start-up „Neuralink“ einen Chip entwerfen, der unter der Haut auf dem Schädelknochen angebracht wird. Die zuvor von einem Roboterchirurgen ins Gehirn gefädelten mikrometerdünnen Fasern leiten diesem Chip Daten zu, die drahtlos in einen hörgeräteähnlichen Minicomputer gelangen, den man per Smartphone und Computer steuern kann. Umgekehrt gelangen so auch Daten und Befehle in das Gehirn. ${ }^{27}$ Ungeahnte Fähigkeiten sollen dem Menschen in Zukunft per Hirnware-Update daraus erwachsen. Aber welche neuen externen Konditionierungen kommen da auf ihn zu? Bereitet man so einem „neuen Denken“ den Weg?

Es kann schnell passieren, dass der Mensch - in technische Regelkreise hinein adaptiert - zum integralen Teil eines Systems wird, in dem er als Person verschwindet. ${ }^{28}$ In dem Augenblick, wo Computertechnologie oder Robotik in seine körperlichen Vollzüge implantiert wird, um bis zu Gehirn und Bewusstsein vorzudringen, ist es schon um ihn geschehen. Der Cyborg ist am Ende nicht mehr menschliche Substanz mit spezifisch menschlichen Vollzügen, sondern ein Agglomerat, ein Bündel von akzidentellen Relationen, die ihn externen Zweckbestimmungen gefügig machen.

27 Vgl. Artikel in der FAZ vom 29.8.2020 Elon Musk präsentiert Neuro-Chip für den Kopf, https://www.faz.net/aktuell/wirtschaft/digitec/neuralinkelon-musk-praesentiert-neuro-chip-fuer-den-kopf-16928167.html (4.9.2020).

28 Vgl. H. Tetens, Der Glaube an die Wissenschaften, 279: „Der (natur) wissenschaftlich vollständig erforschte Mensch wäre der vollkommen gläserne und allen technischen Manipulationen im Prinzip zugänglich gemachte Mensch. Jederzeit bestünde dann die Möglichkeit, ihn zu einer bloß technischen Sache, zu einer beliebig umbaufähigen und perfektionierbaren Biomaschine zu machen. (...) so werden wir eines Weltzustandes ansichtig, in welchem der Mensch jederzeit als moralisch verantwortliche Person abgeschafft und er auf eine technisch beliebig verfügbare Sache degradiert werden könnte." 
Eine fluide Identität der Modi des Menschseins suggeriert uns eine durch eine überstaatliche Agenda forcierte Gendertheorie: Ein rein genetisches Verständnis des Menschen lässt die Ansicht entstehen, was männliche oder weibliche Geschlechtlichkeit ausmache, liege allein in der Variabilität der Chromosomen begründet. Die biologische „Natur“ des Menschen, die phänomenologisch in der Ausprägung der Geschlechtsorgane zum Ausdruck kommt, zählt hier nicht. Sex und Gender stehen einander gegenüber. Letzteres ist ein bloßes gesellschaftliches Konstrukt und ein durch soziale Einflüsse erzeugtes Gefühl. Zugrunde liegt ein nicht offen zutage tretender moderner Cartesianismus. Danach besitzt der Mensch keine vorgegebene leib-seelische Natur, sondern findet sich als rein spirituelles Ich einer körperlichen Verfügungsmasse gegenübergestellt, die er geschlechtlich auch umpolen kann, wenn es beliebt. Mädchen mit einem weiblichen Körper sollen ein Mann werden können, und Jungen mit einem männlichen Körper eine Frau. Da die menschliche Biologie, die den Körper prägt, wesentlicher Bezugspunkt für die Identität des Menschen ist, erweist sich die Behauptung von Genderideologen, das psychologische Gefühl gebe an, welches Geschlecht jemand habe, als irrig. Auch die These, dass es etwas in Deutschland 60 verschiedene Geschlechter gebe, ist zu hinterfragen. Ein falsches Menschenrechtsdenken, nach dem jeder Mensch seine eigene Geschlechtlichkeit müsse wählen können, lässt heute viele Jugendliche, die nicht auf erwachsene Leitung setzten können, in einer fluiden Identität dahinleben. Sie meinen, sich neu erfinden zu müssen, was ihnen auch als "Chance" eingeredet wird.

Die angezeigten Problemüberhänge in der Konfrontation der christlichen Sicht vom Menschen mit der Postmoderne machen deutlich: Für die christliche Anthropologie ergibt sich das Desiderat einer Neubelebung der aristotelischen Philosophie, die bis zur prôte ousia des Menschen vorstößt. Die Ausrichtung an einem Wesensdenken im Zuge einer kritischen Auseinanderset- 
zung mit dem Postmodernismus ist eine erstrangige Herausforderung für das christliche Denken vom Menschen, das die relevanten Einsichten der philosophia perennis zu integrieren hat.

\section{Chrześcijańska antropologia i wyzwania ponowoczesności}

Autor ukazuje główne przesłanki chrześcijańskiej antropologii oraz ich kwestionowanie przez ponowoczesność. Jednym z głównych zagrożeń jest antropomorfizm, gdzie odbiera się człowiekowi jego wyjątkowość w świecie stworzonym. W odróżnieniu od świata zwierzęcego, gdzie rządzi instynkt, człowiek jest duchowo-cielesną osobą, dlatego będąc w świecie, posiada ten świat. Ponowoczesne myślenie ze swoją optyką biotechnologicznego transhumanizmu prowadzi z jednej strony do powstania zmieszanej istoty z biologicznej masy i technologicznego artefaktu. Natomiast $z$ drugiej wspiera ono arbitralny genderyzm, który lekceważy fakt, że ludzka biologia stanowi istotny punkt tożsamości człowieka. 